\title{
BMJ Open Follow Your Virtual Trainer (FYVT): a randomised controlled trial protocol of IT-based lifestyle intervention programme to promote physical activity and health among middle-aged Hong Kong Chinese
}

Stanley Sai-Chuen Hui, ${ }^{1}$ Yao Jie Xie, ${ }^{2}$ Ron Chi-Wai Kwok, ${ }^{3}$ Eric Wing-Cheung Tam, ${ }^{4}$ Winnie Wing Sze Mak, ${ }^{5}$ Phoenix Kit-Han Mo ${ }^{6}$

To cite: Hui SS-C, Xie YJ, Kwok RC-W, et al. Follow Your Virtual Trainer (FYVT): a randomised controlled trial protocol of IT-based lifestyle intervention programme to promote physical activity and health among middle-aged Hong Kong Chinese. BMJ Open 2018;8:e017908. doi:10.1136/ bmjopen-2017-017908

- Prepublication history and additional material for this paper are available online. To view these files, please visit the journal online (http://dx.doi. org/10.1136/bmjopen-2017017908).

Received 26 May 2017 Revised 4 0ctober 2017 Accepted 12 October 2017

Check for updates

For numbered affiliations see end of article.

Correspondence to Professor Stanley SaiChuen Hui; hui2162@cuhk.edu.hk

\section{ABSTRACT}

Introduction Hong Kong is a highly urbanised city where many people work long hours. The limited time and lack of professional instruction are the typical barriers to exercise. The purpose of this study is to test the effectiveness of an information technology-based lifestyle intervention programme on improving physical activity (PA) level and health status in a sample of middle-aged Hong Kong adults.

Methods and analysis A two-arm parallel randomised controlled trial named 'Follow Your Virtual Trainer' will be conducted among 200 physically inactive Chinese adults aged from 40 to 65 years. Those randomly allocated to an intervention group will be under the instruction of a web-based computer software termed 'Virtual Trainer (VT)' to conduct a 3-month self-planned PA programme. A series of online seminars with healthy lifestyle information will be released to the participants biweekly for 3 months. After that, 6 months observation will follow. Those in the control group will only receive a written advice of standard PA recommendation and the textual content of the seminars. The assessments will be implemented at baseline, the 3rd, 6th and 9th months. The primary outcome is PA measured by accelerometer and International Physical Activity Questionnaire. The secondary outcomes include cardiorespiratory fitness, resting energy expenditure, anthropometrics, body composition, blood pressure, health-related quality of life, sleep quality and quantity, fatigue, behaviour mediators and maintenance of PA. The main effectiveness of the intervention will be assessed by a linear mixed model that tests the random effect of treatment on outcomes at the 3rd, 6th and 9th months.

Ethics and dissemination This trial has been approved by the Joint Chinese University of Hong Kong-New Territories East Cluster Clinical Research Ethics Committee (CRE 2015235). The study results will be presented at scientific conferences and published in peer-reviewed journals.

Trial registration number NCT02553980.

\section{Strengths and limitations of this study}

- This is the first study using an online virtual training system for participants to plan and implement their own physical activity (PA) programme for healthy lifestyle improvement. This trial will implement the self-planned personalised PA programme through a human-computer interactive system. The information technology (IT)-based lifestyle intervention is fast, inexpensive, flexible and convenient for adults, especially those with a busy work life.

- The major limitation is from the self-management of IT-based training. No one monitors the participants' PA in real time. The practice compliance depends on the participants' willingness to some extent.

\section{INTRODUCTION}

The benefits of regular physical activity (PA) on health are well documented. The guidelines for practicing the proper amount of exercise in daily life have also been released to the general public. ${ }^{1-4}$ However, $31 \%$ of the world's population is still not physically active enough to meet the recommended minimum level of $\mathrm{PA}^{5}$ The pandemic of physical inactivity thereby should be a public health priority. ${ }^{6} 7$ In Hong Kong, recent cross-sectional surveys reported that as many as $70.9 \%$ (Sport-for-All Survey 2009) to $71.4 \%$ (Community Fitness Survey 2012) of Hong Kong adults were not active enough to reach the guidelines. ${ }^{8} 9$ Although the importance of PA has been widely publicised, more effort is needed to encourage regular PA participation in the Hong Kong population.

The number of middle-aged adults, who are typically ranged from 40 to 65 years of 
age ${ }^{10}$ increased dramatically in Hong Kong in recent years. The 2013 population statistics by the Census and Statistics Department of the Hong Kong Government reported that due to the baby boom in the 1950s and 1960s and an influx of young immigrants during the 1970 and 1980s, the number of people whose ages range from 45 to 64 years significantly increased in the recent 10 years. The proportion almost jumped by $10 \%$ from 2001 $(22.0 \%)$ to $2012(31.4 \%) .{ }^{11}$ Many health problems may occur when the middle-aged adults step into older age. The increasing healthcare cost and aggravating disease burden are serious challenges to an ageing society.

Hong Kong is a highly urbanised city where many people work long hours daily and over the week, especially these middle-aged adults. The limited time and lack of professional exercise instruction are always barriers to their participation in PA. They may be too busy to seek professional advice on how to exercise. Although it is common that exercisers seek personal advice and instruction from personal trainers, the lack of channels and time become excuses for non-exercisers to continue their sedentary lifestyle. The question is then asked as to how feasible PA recommendation and constant reminding and encouragement can be easily accessible at any time and at anywhere. With the advances in information technology (IT), the knowledge of PA can be rapidly disseminated through the internet. ${ }^{12} 13$ The role of 'personal trainer' can also be replaced by a computer program in which guidelines and instructions for PA can be programmed into interactive software and disseminated to users via the World Wide Web. The electronic format of PA guidelines, exercise reminders, motivators, as well as immediate feedback and evaluation can be easily provided through IT. Once users obtain instant feedback on their progress and know their health and fitness improvement, their motivation and willingness to keep participating in PA would be strengthened. Based on these ideas and understanding, we developed a web-based computer software named 'Virtual Trainer (VT)' (www.vt.hk), with several cartoon characters of trainers designed to help the users to implement their personal PA training plans. We then designed a lifestyle intervention programme named 'Follow Your Virtual Trainer (FYVT)' according to the VT system, with the purpose of improving PA level and health status for middle-aged Hong Kong adults.

\section{Aims and hypotheses}

The aim of this study is to evaluate the effectiveness of an IT-based lifestyle intervention programme on improving the PA level and health status in a sample of middleaged Hong Kong Chinese adults. The specific objectives include:

1. To determine the effectiveness of the FYVT programme for increasing PA participation and PA level in Chinese adults.

2. To determine the health benefits of PA promotion by FYVT on cardiorespiratory fitness, resting energy expenditure (REE), body composition, blood pressure, health-related quality of life (HR-QoL), sleep quantity and quality and fatigue.

3 . To examine the role of behaviour mediators in predicting behaviour changes during FYVT programme and their associations with outcomes.

4. To evaluate the maintenance of PA for 6 months during FYVT programme.

Specifically, the following hypotheses will be tested:

1. Chinese middle-aged adults who participate in the lifestyle intervention group will have a greater increase in PA than participants in the control group at the end of the 3-month intervention.

2. The participants in the intervention group will also have a higher PA level than those in the control group at the end of 6-month follow-up.

3. The participants in the intervention group will have a better health status than those in the control group in terms of cardiorespiratory fitness, REE, body composition, blood pressure, HR-QoL, sleep quantity and quality and fatigue at the end of the 3-month intervention and 6-month follow-up.

4. At least $60 \%$ of the participants in the intervention group will keep using online VT system to design their personal exercise plans in the 6-month follow-up period.

\section{METHODS}

\section{Study design}

A two-arm parallel individual level randomised controlled trial is designed according to the SPIRIT (Standard Protocol Items: Recommendations for Interventional Trials) statement. ${ }^{14}$ Participants will be randomly allocated into IT-based intervention group or control group to carry out a 3-month intensive programme, with additional 6 months follow-up, to see the effects of the intervention on improving PA and health status.

\section{Participants}

The target study population will be sedentary Chinese adults who use computers and mobile apps frequently. The inclusion criteria include:

1. aged $40-65$ years;

2. able to understand Cantonese and read Chinese;

3. self-reported inactivity (no habitual exercise experience for at least 6 months);

4. the baseline REE is less than $1.05 \mathrm{kcal} \cdot \mathrm{min}^{-1}$ for men and $0.85 \mathrm{kcal} \cdot \mathrm{min}^{-1}$ for women;

5. reachable over telephone;

6. has basic computer skills;

7. has a smartphone and always surfs internet (at least four times per week) and

8. will not leave Hong Kong for a long time (longer than 2 months) during the study period.

The exclusion criteria are:

1. self-reported history of cardiovascular and pulmonary diseases, neurological disorder, musculoskeletal disorder and osteoarthritis; 
2. receiving medically prescribed diet or PA intervention;

3. blood pressure $\geq 160 / 100 \mathrm{~mm} \mathrm{Hg}$;

4. using of medication that may influence exercise performance;

5. for women, currently pregnant or plan to become pregnant in the next 1 year, and those receiving hormonal therapy.

We will collaborate with local non-governmental organisations in the health and family service fields to recruit participants via advertisements in flyers, surface mails and bulletin boards. The interested subjects are required to fill out an assessment form for preliminary screening eligibility before baseline measurement. The form includes demographics, medical history, exercise habits, exercise eligibility (assessed by modified items from Physical Activity Readiness Questionnaire ${ }^{15}$ ) and other personal information. The preliminary eligible subjects will be further invited to take the REE test and blood pressure measurement. Those that fulfil our study requirements and provide the signed informed consent forms are qualified as eligible participants.

\section{Sample size and power analysis}

From the behavioural risk factor survey conducted by the Department of Health of Hong Kong SAR in 2014, ${ }^{16}$ only $37.4 \%$ of Hong Kong adults (aged 18-64 years) reached the PA level by WHO recommendations. ${ }^{17}$ Based on this proportion, a sample size of 87 is needed in each group to detect a $15 \%$ between-group difference (OR 1.83) in the proportion of participants reporting the PA that meets WHO recommendations after a 3-month intervention (control group rate $40 \%$, intervention group rate $55 \%$, power $=80 \%, \alpha=5 \%$; with a two repeated measurements design). This sample size also has $85 \%$ power to detect a difference of $15 \%$ in the proportion of participants reporting an increase in the high level of PA (control group rate $25 \%$, intervention group rate $40 \%$, power $=80 \%, \alpha=5 \%$ ). The proportion of high level of PA estimated in the calculation is also drawn from the government survey. ${ }^{18}$ With the consideration of $10 \%$ dropout, the final sample size is determined as 100 for each group $(87+87 \times 10 \%=96 \approx 100)$.

\section{Randomisation, concealment and blinding}

The randomisation will be done on the individual level after baseline assessment. Eligible participants will be randomly assigned to lifestyle intervention group or control group with the allocation ratio of 1:1. To ensure allocation concealment, an independent statistician who is not involved in recruitment and baseline assessment will conduct the randomisation by computer-generated allocation sequence. Another researcher will be responsible for arranging the participants to undertake their corresponding treatments; they will not be told which group is the 'true' intervention group, and in both groups they will receive some 'treatments'.

\section{Intervention}

\section{Theoretical model for behaviour change}

The theory of planned behaviour ${ }^{19} 20$ is adopted as the theoretical basis for the behavioural intervention in the FYVY programme. According to this theory model, three types of considerations guide the human behaviours: behavioural beliefs, normative beliefs and control beliefs. The first type is belief in the likely consequences of the behaviour, the second type is beliefs about the normative expectations of others and the third one is those beliefs on the factors that may facilitate or hinder the performance of the behaviour. ${ }^{19} 21$ These considerations emphasise the importance of an individual's intention to regulate their own PA and healthy lifestyle by cultivating a positive attitude and believing it to be within their own control. ${ }^{22}$ Several measures associated with this model are obtained as a matter of course in the study, including fatigue, sleep quality, quality of life and behaviour mediators.

\section{The VT system}

The 'VT' (www.vt.hk), an interactive web-based computer software with the integration of the telecommunication instrument (mobile phone messages and apps), was developed by our research team in 2006 (first version). It had undergone a major modification in 2010 (second version) and is under reconstruction for an enhanced version at present (third version). The VT system provides professional guidelines as well as multilevel electronic motivators for improving PA participation. It likes a traditional personal trainer, but no real physical form of trainer or instructor is needed, which implies a 'VT'. We have designed 16 cartoon characters of the trainer. Users may select any one of them as their personal trainer, and they will have their own personalised web page that simulates an exercise training room (figure 1).

The VT system can provide preliminary online health screening and health-related fitness assessments (Health-fitness Evaluation Module). Then, suggestions of exercise prescriptions will be automatically generated by the VT system (Prescription Module). Moreover, to cater for individual preferences on exercise type, frequency and intensity within the context of personalised exercise prescriptions, the VT system will adopt an interactive approach to retrieve preferences from the users by a series of questions (Scheduler). The system will automatically arrange the preferences of the users, such as date and time of exercise, type and intensity of exercise, etc, into a scheduler programme. The scheduler programme will keep tracking the progress of the actual implementation of the users' exercise. Then feedback about the progress, recommendations for changes, reinforcements and other incentive messages will be generated by VT (Progress Evaluation Module). Each recommended exercise programme will usually last for $2-4$ months, then users will be asked to perform the online health-fitness evaluation again in order to assess their improvement in personal health and fitness. The VT system also includes the frequent and daily dissemination of short messages 


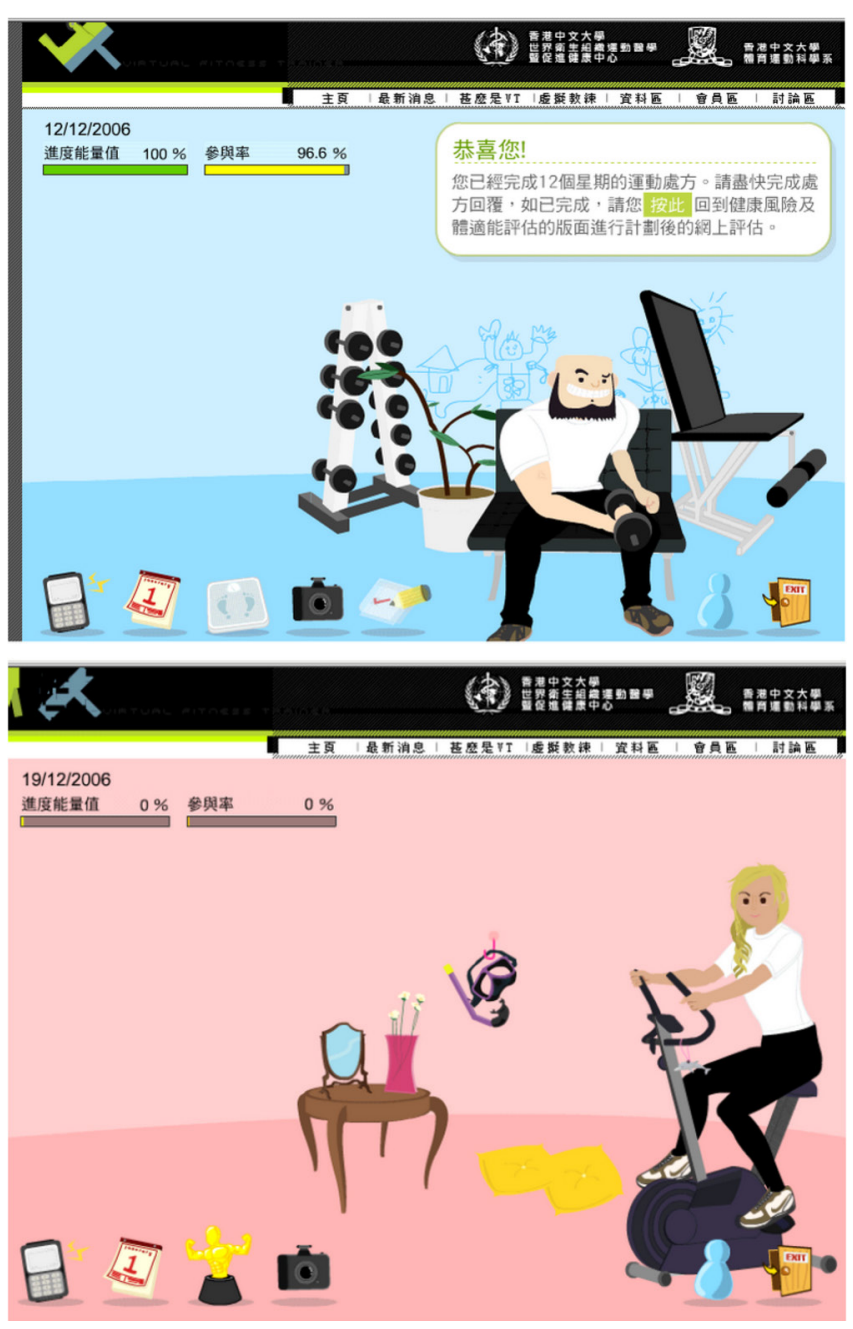

Figure 1 Screenshots of two Virtual Trainer 'rooms'.

to participants via email and short message service (SMS) to their mobile phone. These messages include reminder messages for exercise, incentive messages, positive reinforcement messages, as well as helpful tips on exercise and diet. We are now developing the third version of the VT system to allow accurate PA tracking with the integration of automatic detection and transmission of heart rate and pedometer. All this information will be updated in time through newly designed mobile Apps and transferred to an online system.

\section{Intervention procedures}

Participants in the intervention group will receive 3 months of intensive intervention and 6 months follow-up observation. A briefing session will be conducted at first to introduce the conception of the FYVT programme, the usage of online VT system, the common types of moderate and vigorous $\mathrm{PA}$, the standard duration and frequency of exercise during the intervention, the measurement indicators and other related issues to the participants. After this session, participants are required to design their own 3-month PA plan through a VT system; the basic requirement is no less than two times a week, accumulated through at least $150 \mathrm{~min} /$ week of moderate PA or $75 \mathrm{~min} /$ week of vigorous PA. During these 3 months, six online seminars will be released to the participants in turn and biweekly; the private web-link of these seminars will be sent to their email box and mobile phone. We develop these psychological theme-based seminars that collaborate with a clinical psychologist as well as public health and exercise experts to help the participants to tackle lifestyle risk factors. The contents include introductions of what healthy lifestyles are and how to live healthier, the professional advice on how the appropriate physical activities are beneficial for health, how to integrate appropriate physical activities into daily life, and the strategies for improving exercise compliance and maintenance. The seminar topics include: (1) eating a healthy diet; (2) keeping your weight, waist and blood pressure in check; (3) stopping smoking and cutting back on alcohol consumption; (4) positive psychology on self-management; (5) knowing your physical fitness and (6) more exercise, better life.

Participants randomised to the control group will only receive a written advice of $\mathrm{WHO}$ recommendation of $\mathrm{PA}$ at baseline and receive the text version of six seminars via email, under the same intervals as the intervention group.

\section{Outcomes}

The primary outcome is PA and the secondary outcomes are cardiorespiratory fitness, REE, anthropometrics, body composition, blood pressure, HR-QoL, sleep quality and quantity, fatigue and maintenance of PA. The role of behaviour mediators draw from the theory of planned behaviour will also be examined.

\section{Primary outcomes}

PA will be measured through two ways:

1. Accelerometer (Actigraph GT3X, Florida, USA) for objective PA measurement. The accelerometer can measure the frequency, duration and intensity of PA. It records movement on the vertical and horizontal axis and allows classification of sedentary, light, moderate and vigorous activity levels. ${ }^{23}$ Participants are told to wear the monitor on their right hip by an elastic belt during their waking hours for 7 consecutive days, except when swimming or showering. Sleep time will be recorded on a log sheet. During their wearing time, daily SMS messages will be sent to them to remind them of compliance in wearing. The data contain a minimum of 10 hours/day wear time and 5 of the 7 days are included for analysis. Non-wear time was defined as 60 consecutive zeros (intervals of at least $60 \mathrm{~min}$ ).

2. International Physical Activity Questionnaire (IPAQ) for subjective PA measurement. The Chinese short form version of IPAQ ${ }^{24}$ is used for participants to report their PA during the last 7 days, across all domains of transportation, work, household tasks and leisure time. The duration (in minutes) and frequency (days) of walking, moderate-intensity and vigorous-intensity activity are recorded. The total metabolic equivalents (METs) by the total minutes per 
week of each activity are calculated, resulting in a PA estimation in MET-minutes/week, together with the evaluation for duration and frequency of activities, and three levels of PA (low, moderate and high) can be classified. ${ }^{24}$

\section{Secondary outcomes}

\section{Cardiorespiratory fitness}

The maximal oxygen intake $\left(\mathrm{VO}_{2}\right.$ max in $\left.\mathrm{mL} \cdot \mathrm{min}^{-1} \cdot \mathrm{kg}^{-1}\right)$ is used as an indicator of cardiorespiratory fitness in our study. $\mathrm{VO}_{2}$ max refers to the greatest amount of oxygen that an individual can take in from the inspired air during intense or maximal exercise. ${ }^{25}$ It is considered the best measure of cardiovascular fitness and aerobic endurance. ${ }^{26}$ The $\mathrm{VO}_{2}$ max will be measured using a symptom limited maximal treadmill exercise test in a sports performance laboratory. Under a strict exercise protocol, participants begin the test wearing masks to direct the air into a portable metabolic analyser (Cosmed K4b2, Rome, Italy); oxygen intake is computed each minute as the test proceeds towards the maximal effort. The highest level of oxygen consumed is recorded subsequently.

\section{Resting energy expenditure}

The measurement of REE includes resting oxygen consumption $\left(\mathrm{VO}_{2}\right.$ in $\left.\mathrm{mL} \cdot \mathrm{min}^{-1} \cdot \mathrm{kg}^{-1}\right)$, kilocalorie expenditure $\left(\mathrm{kcal}\right.$ in $\mathrm{kcal} \cdot \mathrm{min}^{-1}$ ) and resting heart rate. These will also be measured by the metabolic analyser (Cosmed K4b2). Participants are asked to wear the analyser and lie on a bed for $20 \mathrm{~min}$, the temperature and humidity of the laboratory will be adjusted to a comfortable situation. $\mathrm{VO}_{2}$ and kilocalorie expenditure are measured breath-by-breath, and the lowest continuous $10 \mathrm{~min}$ of metabolic values are recorded. The heart rate is measured after $20 \mathrm{~min}$ rest.

\section{Anthropometrics and body composition}

The measurements include weight, height, waist circumference, hip circumference, percentage of body fat and body mass. A trained research assistant will conduct all the measurement bases on the standard protocol. ${ }^{27}$ All measures will be conducted two times and the mean value of two measurements is calculated. The anthropometrics are measured to the nearest $0.1 \mathrm{~kg}$ or $\mathrm{cm}$ where appropriate. Body mass index (BMI) is calculated as weight divided by the square of height $\left(\mathrm{BMI}=\mathrm{kg} / \mathrm{m}^{2}\right)$. Obesity is defined as BMI $\geq 25 \mathrm{~kg} / \mathrm{m}^{2}$ according to WHO standard for Asian populations. ${ }^{28}$ A bioelectrical impedance analysis $^{29}$ (Tanita, BC 581, Tokyo, Japan) will be used to test body fat percentage. Fat mass and lean mass are calculated subsequently.

\section{Blood pressure}

Blood pressure will be measured through mercury sphygmomanometer by a clinical professional staff under the guideline of standard protocol. ${ }^{30}$ After at least $5 \mathrm{~min}$ sitting, measurement is taken on the right arm of the participant; an appropriately sized cuff is used. Every participant will be measured two times, and the mean of the two measurements is computed.

\section{Health-related quality of life}

A Chinese (HK) version ${ }^{31}$ of the Medical Outcomes Study 36-item Short Form Health Survey (SF-36) is used for HR-QoL assessment. The SF-36 is a widely used instrument to assess HR-QoL in eight dimensions $^{32}{ }^{33}$ : physical functioning, role physical, bodily pain, role emotional, social functioning, mental health (MH), vitality (VT) and general health perception. Two summary scores, namely, the physical health summary (PHS) and mental health summary (MHS), can be calculated from the eight dimension scores. It is an overall assessment of the quality of life in terms of physical and MH status. The PCS and MCS have been identified as valid and equivalent in the Chinese population in Hong Kong. ${ }^{34}$

\section{Sleep quality and quantity}

Sleep quality will be measured by the Chinese version of the Pittsburgh Sleep Quality Index (PSQI) ${ }^{35}$ The 19-item PSQI assesses seven dimensions of sleep quality over the past month: sleep latency and duration, subjective sleep quality, sleep disturbances, habitual sleep efficiency, use of sleep medication and daytime dysfunction. Score $0-3$ is used for each dimension, and a cumulative score (0-21) can be calculated by adding the seven dimensions' scores. Higher scores indicate poorer sleep quality. ${ }^{36}$ Sleep quantity will be measured by a 7 -day daily sleep log. ${ }^{35}$ Participants are asked to record their sleep status for 7 consecutive days when they wake up in the mornings. The sleep log collects information on time to go to bed, sleep-onset latency, waking time in the morning and frequency of awakenings by the corresponding four questions: (1) I went to bed at ____ (clock time) yesterday evening; (2) I fell asleep in ____ (minutes) yesterday evening; (3) I got out of bed at ____ (clock time) this morning and (4) I woke up during the night ____ times. ${ }^{35}$ The total sleep time can thus be calculated as the interval time (minutes) between going to bed and waking up in the morning minus the time spent in falling asleep.

\section{Fatigue}

The Numeric Rating Scale-fatigue ${ }^{37}$ is used to evaluate fatigue level. It is a scale with numbers from 0 to 10 for participants' self-rating. The score ranges from 0 to 10 , in which 0 presents no fatigue at all and 10 presents the highest experience of fatigue. A higher score indicates that heavier fatigue is experienced.

\section{Maintenance of PA}

To examine the maintenance of PA after the cessation of the 3-month intensive programme, a self-administered questionnaire that includes IPAQ will be given to the participants at 6 and 9 months during the follow-up period. We will determine the maintenance mainly in two aspects: (1) what percentage of the participants in the intervention group keeps using online VT system to design their personal exercise plans for at least 1 month 
in the 6-month follow-up period and (2) in what percentage of the 24 weeks in the 6-month follow-up period are the participants in the two groups regularly active at a moderate intensity?

\section{Behaviour mediators}

The possible behaviour mediators will be examined by a self-designed questionnaire (online supplementary appendix I) that is based on Ajzen's guideline for developing a Theory of Planned Behaviour Questionnaire. ${ }^{21} \mathrm{We}$ will structure these mediator variables according to attitude, subjective norms and perceived behavioural control, to understand their intention towards active participation in physical activities. A seven-point scale is used for each item in the questionnaire. A Higher score indicates the higher influence of the mediator on behaviour change.

\section{Procedure of assessment and follow-up}

After screening for eligibility, the eligible participants are required to take the baseline measurement. Since the accelerometer needs 7 days wear time, we ask the participants to wear the monitor for 7 days first, and then come to our laboratory again for other baseline measures. Randomisation is taken subsequently. Figure 2 demonstrates the overview of protocol procedure. The follow-up assessments will be conducted at the end of the 3rd, 6th and 9th month after the trial begins. The research assistant will regularly monitor the progress of PA of each participant through the VT system. If there is a delay or missing of PA training during the intervention period, he will send reminders to the corresponding participants through the VT system and help them to catch up on the progress. An event report sheet will be used to record the adverse events by the research assistant every 2 weeks. Table 1 shows the schedule of each outcome measurement. Participants are required to wear an accelerometer 7 days prior to answering the IPAQ to ensure the time interval of PA measured by the accelerometer is in accordance with that measured by the IPAQ (recall last 7 days' PA).

\section{Statistical analysis}

Double data entry will be adopted. Multiple imputations will be used for the missing data. Data will be analysed both by the intention-to-treat (ITT) principle (include participants who have valid baseline assessments, regardless of whether they drop out later) and by the completed case analysis (only those who participate in

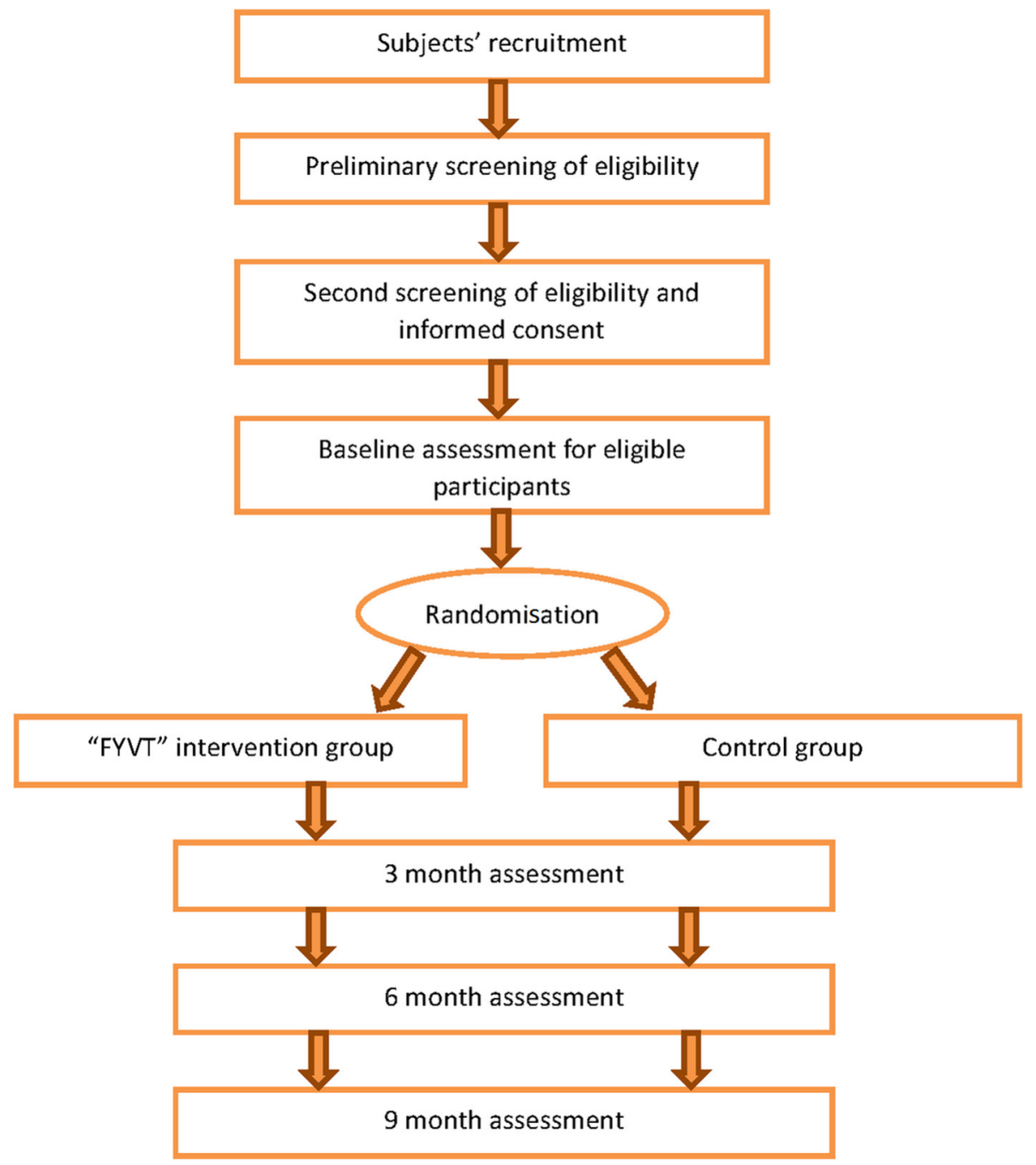

Figure 2 Procedure of screening eligibility, assessment and follow-up. FYVT, Follow Your Virtual Trainer. 
Table 1 Procedures of recruitment screening and outcome measurements

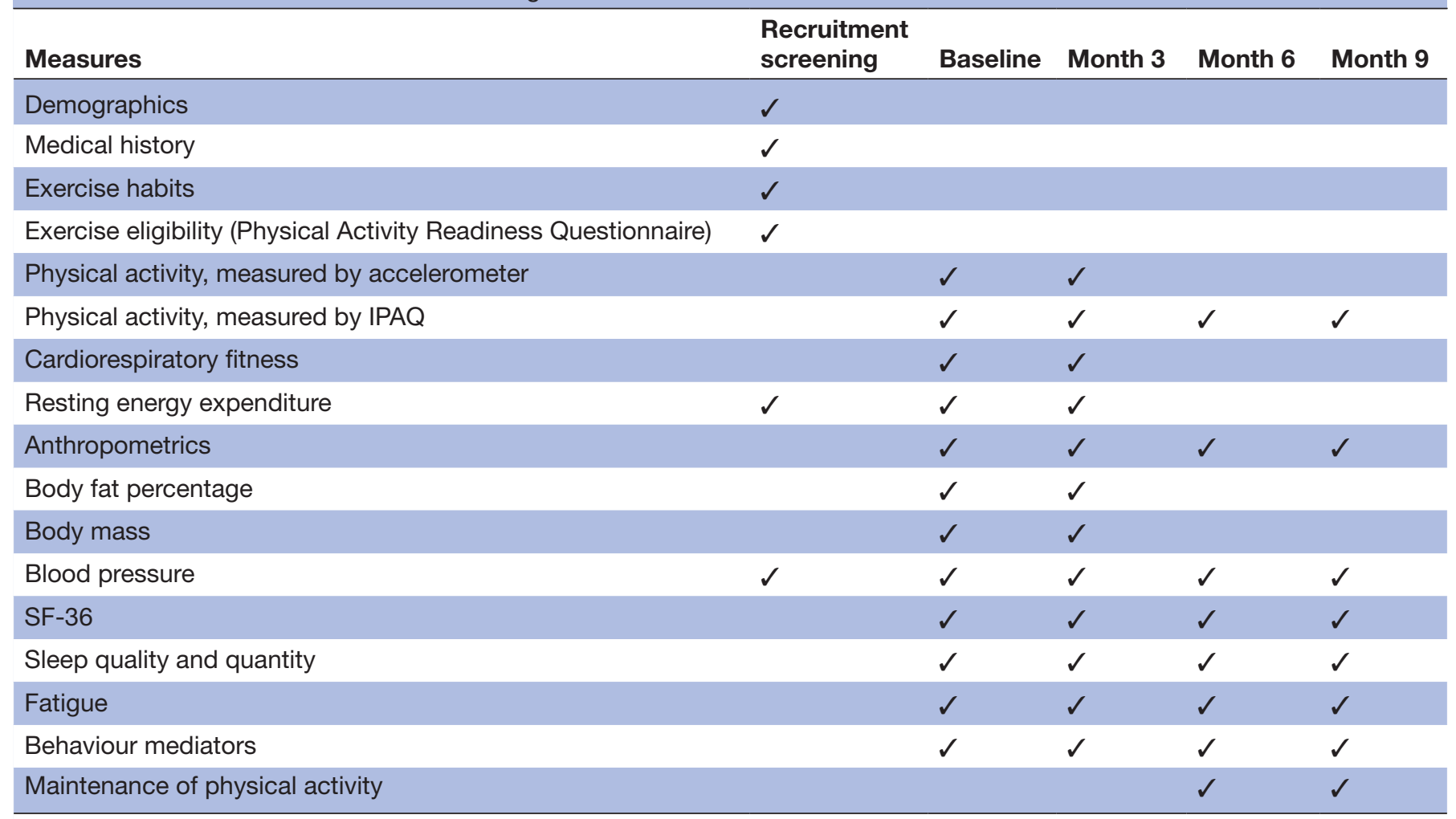

IPAQ, international physical activity questionnaire; SF-36, 36-item short form health survey.

the programme for the full period are involved). If the results from two analytical approaches are similar and the dropout rate is less than $10 \%$ in two groups, ITT results will be adopted. One-way analysis of variance and Pearson $\chi^{2}$ test are used to compare baseline differences between two groups for continuous variables (age, METs, $\mathrm{VO}_{2}$ max, REE, anthropometrics and body composition, blood pressure, HR-QoL, sleep quality and quantity, fatigue and behaviour mediators) and categorical variables (PA level, obesity and categorical demographic variables), respectively. The effectiveness of the intervention will be assessed by the linear mixed model, which tests the random effect of treatment on outcomes at the 3rd, 6th and 9th months when measured as mean, as well as tests the trend of changes by taking four measurement time points (baseline, 3rd, 6th, 9th months) as a random effect in the model. Logistical regression will be used and OR is calculated to examine the effect of the intervention on categorical variables. The associations between behaviour mediators and changes in outcomes will be examined by multiple linear regression analysis.

\section{Ethics and dissemination}

All participants shall provide written informed consent (online supplementary appendix II) to the investigators before the baseline assessment. All the data are confidential and only used for research purpose. Only the researchers who get the permission from the principal investigator have the right to access to the data. The trial was registered at http:/ / www.clinicaltrials.gov with identification no. NCT02553980.

We will disseminate the study results by peer-reviewed publications and conference presentations according to the CONSORT statement recommendations.

\section{DISCUSSION}

Middle-aged adults often show visible signs of ageing such as loss of skin elasticity and greying of the hair. The reduction in aerobic performance and decrease in maximal heart rate also occur as a sign of ageing. Strength and flexibility also decrease throughout middle age. Many health problems may occur when the middle-aged adults step into older age. As the age composition of the worldwide population is altering as median ages rise with an increase in the number of elders, ${ }^{38}$ the increase of disease burden and extra heavy cost for healthcare is becoming a big challenge to modern societies. ${ }^{39}$ Thus, keeping an optimal health status and effectively preventing diseases in middle age are critical for healthy ageing and health policy-making in an ageing society to help the ageing population to maintain positive social engagement and productivity, resulting in a sustained sense of well-being and an extended period of good health for most elderly.

Most people agree that PA is good for health but need constant reminding and encouragement. For the Hong Kong middle-aged adults, the busy work schedule and heavy burden of everyday life make them have less leisure 
time to devote to exercise. To tackle these concerns, the FYVT programme will improve the PA engagement among middle-aged adults and constantly remind them of the need and benefits of PA as well as provide professional advice on exercise prescription and healthy lifestyle. The services provided by the FYVT programme has no time and venue limitations, which greatly enhances the efficiency of dissemination.

The VT system provides knowledge, skills and online demonstration on how to perform PA effectively; it also includes online health-fitness assessment and diet analysis. The VT system enables users to plan and design their individualised PA programme and fit it into a personalised scheduler; then the system will automatically send a reminder message and PA implementation to the users through smartphone apps and email. This is how telecommunication and the internet come into play. The cost is relatively low. This module enables users to plan their own PA programme, which is a typical example of employing the theory of planned behaviour. ${ }^{19} 20$

Although there are many advantages of using an online human-computer interactive system to conduct a lifestyle intervention, our study also has inevitable limitations. Since it is a self-managed virtual training programme, no one monitors the participants' PA in real time. It is a challenge to quality control, which may depend on the participants' willingness of compliance to some extent. Also, the frequency, duration and intensity of PA may be varied among participants in the intervention group. But our major concern is to see whether the IT-based online system can inspire the individuals' interests in PA and improve their positive behaviour. If the effectiveness of this approach is affirmative, this virtual training model can be widely used and benefit more people. Thus, we suggest that the rigour dosage of intervention is not a major problem as long as the participants achieve the basic intensity of PA required in the study. Another limitation is that there might be measurement bias of some self-reporting outcomes, such as fatigue and sleep quality. But we do not think it will overturn the study results because the self-reporting biases are random in both groups.

In summary, it would be an inexpensive, convenient, fast and sustainable approach for adults, especially those with a busy work life, to use an online virtual training system to plan and implement their own PA programme and comply with a healthy lifestyle. If the intervention proves to be effective, it will provide a scientific rationale for the implementation of the self-planned personalised PA programme through a human-computer interactive system.

\footnotetext{
Author affiliations

${ }^{1}$ Department of Sports Science and Physical Education, The Chinese University of Hong Kong, Shatin, Hong Kong, China

${ }^{2}$ School of Nursing, The Hong Kong Polytechnic University, Kowloon, Hong Kong, China

${ }^{3}$ Department of Information Systems, City University of Hong Kong, Kowloon, Hong Kong, China
}

${ }^{4}$ Department of Health Technology and Informatics, The Hong Kong Polytechnic University, Kowloon, Hong Kong, China

${ }^{5}$ Department of Psychology, The Chinese University of Hong Kong, Shatin, Hong Kong, China

${ }^{6}$ The Jockey Club School of Public Health and Primary Care, The Chinese University of Hong Kong, Shatin, Hong Kong, China

Contributors Study concept and design: SS-CH, YJX, RC-WK; Obtained funding: SS-CH, WWSM, PK-HM, YJX; Administrative, technical or material support: SS-CH, EW-CT, WWSM, PK-HM; Drafting of the manuscript: YJX, SS-CH; Critical revision of the manuscript: YJX, SS-CH. All authors read and approved the final manuscript.

Funding This study was supported by the Knowledge Transfer Project Fund from The Chinese University of Hong Kong (KPF14ICF14).

Competing interests None declared.

Patient consent Obtained.

Ethics approval The Joint Chinese University of Hong Kong-New Territories East Cluster Clinical Research Ethics Committee.

Provenance and peer review Not commissioned; externally peer reviewed.

Open Access This is an Open Access article distributed in accordance with the Creative Commons Attribution Non Commercial (CC BY-NC 4.0) license, which permits others to distribute, remix, adapt, build upon this work non-commercially, and license their derivative works on different terms, provided the original work is properly cited and the use is non-commercial. See: http://creativecommons.org/ licenses/by-nc/4.0/

(c) Article author(s) (or their employer(s) unless otherwise stated in the text of the article) 2018. All rights reserved. No commercial use is permitted unless otherwise expressly granted.

\section{REFERENCES}

1. Department of Health and Human Services. Physical activity and health: a report of the surgeon genereal. Collingdale, Pennsylvania: DIANE Publishing, 1996.

2. Physical Activity Guidelines Advisory Committee. Physical activity guidelines advisory committee report, 2008. . Washington, DC: US Department of Health and Human Services, 2008:2008. A1-H14.

3. Shiroma EJ, Lee IM. Physical activity and cardiovascular health: lessons learned from epidemiological studies across age, gender, and race/ethnicity. Circulation 2010;122:743-52.

4. Haskell WL, Lee IM, Pate RR, et al. Physical activity and public health: updated recommendation for adults from the American College of Sports Medicine and the American Heart Association. Med Sci Sports Exerc 2007;39:1423-34.

5. Hallal PC, Andersen LB, Bull FC, et al. Global physical activity levels: surveillance progress, pitfalls, and prospects. Lancet 2012;380:247-57.

6. Kohl HW, Craig CL, Lambert EV, et al. The pandemic of physical inactivity: global action for public health. Lancet 2012;380:294-305.

7. Lee IM, Shiroma EJ, Lobelo F, et al. Effect of physical inactivity on major non-communicable diseases worldwide: an analysis of burden of disease and life expectancy. Lancet 2012;380:219-29.

8. Leisure and Cultural Services Department H. A Report for the Consultancy Study on Sport for All -Participation Patterns of Hong Kong People in Physical Activities. Hong Kong: Leisure and Cultural Services Department, HKSAR and the Department of Sports Science and Physical Education, The Chinese University of Hong Kong, 2009.

9. Leisure and Cultural Services Department H. A report for the consultancy study on physical fitness test for the community 2011. Hong Kong: Leisure and Cultural Services Department, HKSAR, and the Department of Sports Science and Physical Education, The Chinese University of Hong Kong, 2012.

10. Shephard RJ. Aging, physical activity, and health. Champaign, Illinois: Human Kinetics Publishers, 1997.

11. Social Statistics Branch, Census and Statistics Department, HKSAR Women and men in Hong Kong: key statistics. 2013. https://www. censtatd.gov.hk/hkstat/sub/sp180.jsp?productCode=B1130303 2013

12. Vandelanotte C, Spathonis KM, Eakin EG, et al. Website-delivered physical activity interventions a review of the literature. Am J Prev Med 2007;33:54-64.

13. Christakis NA, Fowler JH. Connected: the surprising power of our social networks and how they shape our lives. New York: Hachette Digital, Inc., 2009. 
14. Chan AW, Tetzlaff JM, Gøtzsche PC, et al. SPIRIT 2013 explanation and elaboration: guidance for protocols of clinical trials. BMJ 2013;346:e7586.

15. Warburton DE, Jamnik VK, Bredin SS, et al. The Physical Activity Readiness Questionnaire for Everyone (PAR-Q+) and electronic Physical Activity Readiness Medical Examination (ePARmed-X+). Health Fit J Canada 2011;4:3-17.

16. Surveillance and Epidemiology Branch, Centre for Health Protection of the Department of Health. Level of physical activity by $\mathrm{WHO}$ recommendations. 2012. http://www.chp.gov.hk/en/data/1/10/280/ 1341.html

17. World Health Organization. Global recommendations on physical activity for health. 2010. http://apps.who.int/iris/handle/10665/ 44399? locale=en

18. Surveillance and Epidemiology Branch, Centre for Health Protection of the Department of Health. Level of physical activity. 2012. http:// www.chp.gov.hk/en/data/1/10/280/1364.html

19. Ajzen I. The theory of planned behaviour: reactions and reflections. Psychol Health 2011;26:1113-27.

20. Ajzen I. The theory of planned behavior. Organ Behav Hum Decis Process 1991;50:179-211.

21. Ajzen I. Constructing a theory of planned behavior questionnaire. https://people.umass.edu/aizen/pdf/tpb.measurement.pdf

22. Courneya KS, McAuley E. Cognitive mediators of the social influence-exercise adherence relationship: a test of the theory of planned behavior. J Behav Med 1995;18:499-515.

23. Trost SG, Mclver KL, Pate RR. Conducting accelerometer-based activity assessments in field-based research. Med Sci Sports Exerc 2005;37:S531-43.

24. Macfarlane DJ, Lee CC, Ho EY, et al. Reliability and validity of the Chinese version of IPAQ (short, last 7 days). J Sci Med Sport 2007;10:45-51.

25. Anon. Quantitative exercise testing for the cardiac patient: the value of monitoring gas exchange. Atlanta, Georgia, March 7-8, 1986. Proceedings. Circulation 1987;76:VI1-58.

26. Fletcher GF, Balady GJ, Amsterdam EA, et al. Exercise standards for testing and training: a statement for healthcare professionals from the American Heart Association. Circulation 2001;104:1694-740.
27. Norton K, Olds T. Anthropometrica: a textbook of body measurement for sports and health courses. Kensington, NSW: UNSW Press, 1996.

28. World Health Organisation, International Association for the Study of Obesity, International Obesity TaskForce. The Asia-Pacific Perspective: redefining obesity and its treatment. Sydney: Health Communications, 2000.

29. Segal KR, Van Loan M, Fitzgerald PI, et al. Lean body mass estimation by bioelectrical impedance analysis: a four-site crossvalidation study. Am J Clin Nutr 1988;47:7-14.

30. Xie YJ, Ho SC, Liu ZM, et al. Comparisons of measured and selfreported anthropometric variables and blood pressure in a sample of Hong Kong female nurses. PLoS One 2014;9:e107233.

31. Lam CL, Gandek B, Ren XS, et al. Tests of scaling assumptions and construct validity of the Chinese (HK) version of the SF-36 Health Survey. J Clin Epidemiol 1998;51:1139-47.

32. Ware JE, Sherbourne CD. The MOS 36-item short-form health survey (SF-36). I. Conceptual framework and item selection. Med Care 1992;30:473-83.

33. Ware JE, Gandek B. Overview of the SF-36 Health Survey and the International Quality of Life Assessment (IQOLA) Project. J Clin Epidemiol 1998;51:903-12.

34. Lam CL, Tse EY, Gandek B, et al. The SF-36 summary scales were valid, reliable, and equivalent in a Chinese population. J Clin Epidemiol 2005;58:815-22.

35. Tsai PS, Wang SY, Wang MY, et al. Psychometric evaluation of the Chinese version of the Pittsburgh Sleep Quality Index (CPSQI) in primary insomnia and control subjects. Qual Life Res 2005;14:1943-52.

36. Buysse DJ, Reynolds CF, Monk TH, et al. The pittsburgh sleep quality index: a new instrument for psychiatric practice and research. Psychiatry Res 1989;28:193-213.

37. Williamson A, Hoggart B. Pain: a review of three commonly used pain rating scales. J Clin Nurs 2005;14:798-804.

38. Department for Economic and Social Affairs. World population prospects, the 2012 revision. New York: Department for Economic and Social Affairs, 2013.

39. Harper S. Economic and social implications of aging societies. Science 2014;346:587-91. 